\title{
Study on Tongue Coating Microbiota in Patients with Atrophic Gastritis
}

\author{
Lingshan Bao, Yingcong $\mathrm{Yu}^{*}$ \\ Department of Gastroenterology, Wenzhou No. 3 Clinical Institute Affiliated to Wenzhou Medical University, Wenzhou People's \\ Hospital, Wenzhou, China \\ Email: *yuyingcong001@126.com
}

How to cite this paper: Bao, L.S. and $\mathrm{Yu}$, Y.C. (2020) Study on Tongue Coating Microbiota in Patients with Atrophic Gastritis. Advances in Microbiology, 10, 681-690. https://doi.org/10.4236/aim.2020.1012049

Received: November 20, 2020

Accepted: December 25, 2020

Published: December 28, 2020

Copyright (c) 2020 by author(s) and Scientific Research Publishing Inc. This work is licensed under the Creative Commons Attribution International License (CC BY 4.0).

http://creativecommons.org/licenses/by/4.0/

\begin{abstract}
Aims: To explore the tongue coating microbiota composition in patients with atrophic gastritis by using the $16 \mathrm{~S}$ rRNA gene sequencing technology. Methods: The study included 29 atrophic gastritis patients and 29 age and gender-matched non-atrophic gastritis controls. By sequencing the V3-V4 region of the 16S rRNA, we investigated the microbial community structure and diversity on the tongue coating. Results: There was no significant difference in the microbial diversity on the tongue coating between the two groups. However, compared with the control, the atrophic gastritis group had a smaller number of operational taxonomic units (OTUs). At the class level, patients with atrophic gastritis had lower relative abundances of Betaproteobacteria and Spirochaetia than the control group. At the gene level, the abundance of Neisseria and Aggregatibacter in atrophic gastritis group had significantly decreased compared with control ones. Furthermore, functional prediction revealed that 24 metabolic pathways significantly differed between the two groups. Conclusions: Our findings provide novel evidence that tongue coating microbiota may be a biomarker for characterizing patient with atrophic gastritis, but its mechanism needs to be further elaborated.
\end{abstract}

\section{Keywords}

Atrophic Gastritis, Tongue Coating Microbiota, 16S rRNA Sequencing

\section{Introduction}

The human is a complex and diverse ecosystem consisting of trillions of microbes (i.e. bacteria, archaea, fungi, protists and viruses) [1], the majority of which are essential in human development, and the complex interactions between the human and the microbes confer benefits to the host in many key aspects of life 
[2]. The microbes are thought to be the second genome of the human body [3], as the human gut microbes alone have up to 3 million genes, 150 times the number of human genes, whose function is not just helpful in physiologic activities like digestion, absorption, protection, growth but also plays an important role in human health and disease through participating in various biochemical pathways [4] [5]. So far, the microbes have been implicated to be closely related to many chronic diseases, such as obesity [6], malignancies [7], diabetes [8] and so on.

Atrophic gastritis (AG) is a relatively common disease globally, the incidence rate of which ranged from $0 \%$ to $10.9 \%$ [9], characterized by the replacement of the original gastric glands by intestinal metaplasia (IM), pseudopyloric metaplasia, and/or fibrosis [10]. As the human model of gastric carcinogenesis with the following sequential stages: chronic gastritis $\rightarrow$ atrophy $\rightarrow$ intestinal metaplasia $\rightarrow$ dysplasia is increasingly accepted [11], the early diagnosis and treatment of atrophic gastritis are of great significance. It has been confirmed that the bacterium is the cause of gastritis in an overwhelming majority of the cases because of the discovery of the Helicobacter pylori $(\mathrm{Hp})$ by Warren and Marshall in 1982 [12] and from current knowledge, the pathogenic mechanisms between bacterium and gastritis may involve in exacerbating gastric mucosal inflammation, altering the host immune system, and promoting the transformation of dietary nitrate carcinogens [13] [14] [15]. However, most of the current studies focus on the gastrointestinal flora, and the research on the relationship between tongue coating bacteria and atrophic gastritis is still in its infancy.

As it is determined that factors including age, gender can significantly alter the composition of the microbiota [1] [16], this study was conducted in patients with atrophic gastritis and non-atrophic gastritis matched with their gender and age. In the present study, we characterized the tongue coating microbiota to evaluate the differences of tongue coating microbiota in patients with atrophic gastritis and non-atrophic gastritis by using the $16 \mathrm{~S}$ rRNA gene sequencing technology.

\section{Materials and Methods}

\subsection{Subject}

The 29 atrophic gastritis patients confirmed by endoscopy and pathology at the Department of Gastroenterology of Wenzhou People's Hospital in Zhejiang, from May 2019 to November 2019 were recruited as cases. In the same period, we included 29 non-atrophic gastritis patients matched with age $( \pm 3)$ and gender of atrophic gastritis patients as controls. All participants were briefed on the purpose, procedures, potential risks and benefits of the study, and informed consent was signed and those who had taken antibiotics or microecological modulators within 2 weeks before were also ruled out. The study was approved by the Ethical Committee of Wenzhou People's Hospital.

\subsection{Sample Collections}

Participants were not allowed to eat or drink before this procedure. A sterile 
swab was used to gently scrape the tongue coating on the patient for 5 - 6 times with standard operations. After collection, the specimen was placed in a sterile tube, transported to the laboratory with liquid nitrogen and stored at $-80^{\circ} \mathrm{C}$ freezing storage until further processing.

\subsection{S rRNA Gene Sequence Analysis}

The 16S rRNA sequencing [17] analysis was used to determine the tongue coating microbiota. The microbial DNA was extracted using the MiSeq Reagent Kit v3 (Illumina, USA). Agarose gel electrophoresis and Nanodrop 2000 (Thermo Fisher Scientific, USA) were used to determine the integrity of the genomic DNA and PCR amplification was carried out in an ABI 2720 Thermal Cycler (Thermo Fisher Scientific, USA). We used isolated DNA as the template for amplification of the V3-V4 hypervariable region of the 16S rRNA gene in three replicate reactions with the primer pair $\mathrm{F}$ (Illumina adapter sequence $1+$ CCTACGGGNGGCWGCAG) and $\mathrm{R}$ (Illumina adapter sequence $2+$ GACTACHVGGGTATCTAATCC). The replicate PCR products were pooled and purified with Agencourt AMPure XP magnetic beads (Beckman Coulter, USA).

\section{4. cDNA Library Construction and Sequencing}

According to the preliminary quantitative results of agarose gel electrophoresis, the Qubit was used to accurately quantify the library. Insert length and integrity of the libraries were assessed by Agilent 2100 bioanalyzer (Agilent Technologies, USA) to check the quality of the library. Later, the library was sequenced by two-terminal sequencing strategy of $2 \times 250$ bp on Miseq platform according to standard protocols [18].

\subsection{Statistical Analysis}

All statistics were analyzed by the SPSS v25.0 (SPSS Inc., Chicago, IL) and R v3.0 (The R Foundation for Statistical Computing, Vienna, Austria) and all measurement data were presented as mean \pm standard deviation. Paired t-test was used to analyze the participant characteristics between the two groups. The relative abundances in each sample were calculated on the basis of the sum of $16 \mathrm{~S}$ rRNA sequences. Furthermore, the Alpha-and beta-diversity were estimated between the two groups using the $\mathrm{R}$ software. A value of $p<0.05$ was considered to be significant in this work.

\section{Results}

In this study, 29 patients with atrophic gastritis including 16 males and $13 \mathrm{fe}-$ males and 29 non-atrophic gastritis matched according to gender and age $( \pm 3)$, were included in the study and the average age was $46.91 \pm 8.41$ years old. Differences in variables, such as gender, age, smoking, alcohol drinking, BMI and the presence $\mathrm{Hp}$ did not achieve significance $(P>0.05)$ between the two groups 
(Table 1).

\subsection{Diversity of the Microbial Community between Two Groups}

A total of 1000 operational taxonomic units (OTUs) were quantified in this study. The Venn diagram of the diversity showed that 695 OTUs were shared among the two groups, 106 OTUs were detected only in atrophic gastritis group and 199 OTUs were only quantified in the controls (Figure 1).

Chao1, abundance-based coverage estimator (ACE), Shannon and Simpson are the comprehensive indexes to reflect the alpha diversity of the microbial community in the tongue coatings. Chaol and ACE reflect community richness, the trend of which was similar to that of OTUs. Shannon and Simpson indexes reflect community diversity. As listed (Table 2), there was no significant difference $(P>0.05)$ in microbial community diversity between the two groups.

Table 1. Characteristics of the two groups in the study.

\begin{tabular}{cccc}
\hline & $\begin{array}{c}\text { Atrophic gastritis } \\
\text { group }(\mathrm{n}=29)\end{array}$ & $\begin{array}{c}\text { Control group } \\
(\mathrm{n}=29)\end{array}$ & $\mathrm{P}$ \\
\hline Male/Female, $\mathrm{n}(\%)$ & $16 / 13(50 \%)$ & $16 / 13(50 \%)$ & 1.000 \\
Age (years; means \pm SD) & $46.97 \pm 8.10$ & $46.86 \pm 8.98$ & 0.736 \\
Smoking, n (\%) & $12(41.38 \%)$ & $6(20.69 \%)$ & 0.089 \\
Drinking, n (\%) & $10(34.48 \%)$ & $6(20.69 \%)$ & 0.240 \\
BMI (kg/m², means \pm SD) & $22.90 \pm 2.77$ & $23.32 \pm 2.91$ & 0.484 \\
$\mathrm{Hp}+/-, \mathrm{n}(\%)$ & $12 / 16(44.83 \%)$ & $6 / 23(20.69 \%)$ & 0.072 \\
\hline
\end{tabular}

Table 2. The alpha diversity of two groups.

\begin{tabular}{cccc}
\hline & $\begin{array}{c}\text { Atrophic gastritis group } \\
(\mathrm{n}=29)\end{array}$ & $\begin{array}{c}\text { Control group } \\
(\mathrm{n}=29)\end{array}$ & $\mathrm{P}$ \\
\hline Chao1 (mean $\pm \mathrm{SD})$ & $346.05 \pm 41.32$ & $357.42 \pm 49.29$ & 0.387 \\
ACE (mean $\pm \mathrm{SD})$ & $347.30 \pm 42.49$ & $360.19 \pm 46.52$ & 0.233 \\
Shannon (mean $\pm \mathrm{SD})$ & $3.47 \pm 0.22$ & $3.45 \pm 0.17$ & 0.477 \\
Simpson (mean $\pm \mathrm{SD})$ & $0.06 \pm 0.02$ & $0.06 \pm 0.01$ & 0.951 \\
\hline
\end{tabular}

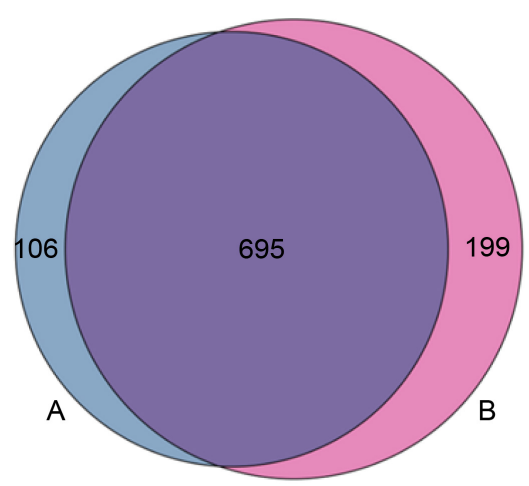

Figure 1. A Venn diagram showing the comparison of microbiota diversity between the two groups by at the OTUs level. A: Atrophic gastritis group; B: Control group. 


\subsection{Relative Abundances of Microbes}

The relative abundances of bacteria at the class level in each of the two groups was produced (Figure 2). Dominant bacteria of tongue coating $(5 \% \geq$ relative abundance) in the two groups were similar and the greatest relative abundances were Bacteroidia, Bacilli, Betaproteobacteria, Fusobacteriia, Negativicutes, Actinobacteria, Gammaproteobacteria, Clostridia. Moreover, patients with atrophic gastritis had lower relative abundances of Betaproteobacteria and Spirochaetia than the control group $(P<0.05)$ (Figure 3$)$.

At the gene level, the dominant bacteria in the two groups were basically the same and relative abundance was slightly different. The abundance of atrophic

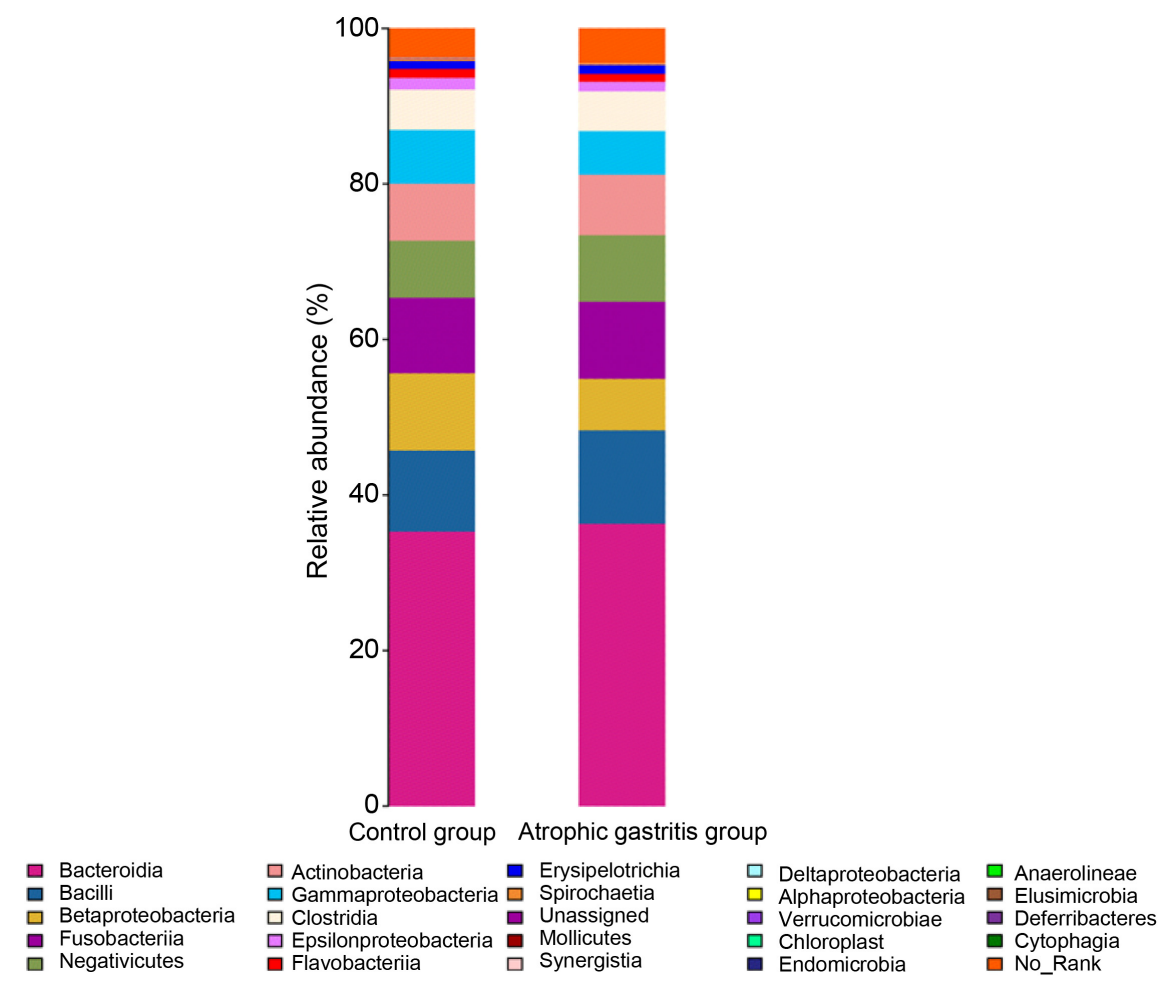

Figure 2. The relative abundances of bacteria at the class level in two groups.

Betaproteobacteria*

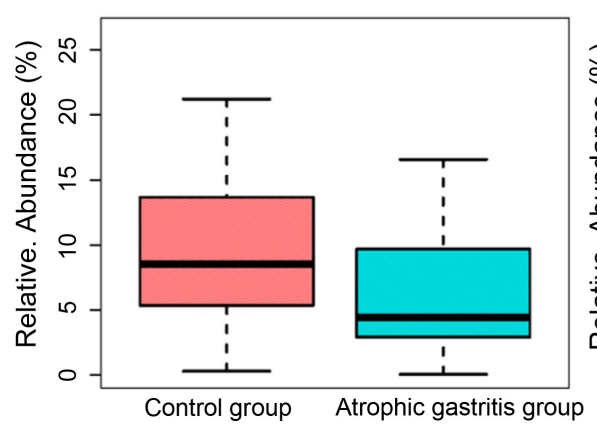

Spirochaetia*

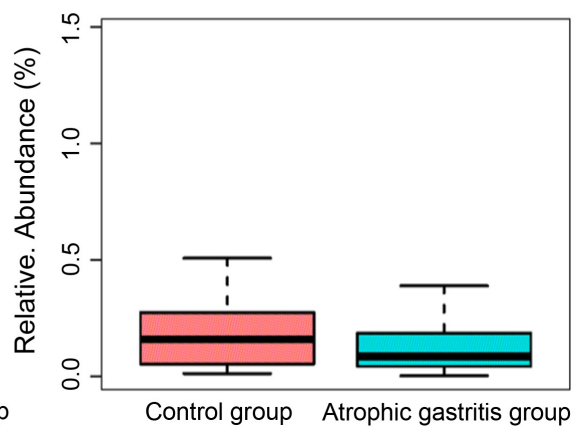

Figure 3. Relative abundance of Betaproteobacteria and Spirochaetia in two groups. $\left({ }^{*}=P<\right.$ $0.05)$. 
gastritis group from high to low were: Prevotella (23.95\%), Streptococcus (8.16\%), Alloprevotella (6.97\%), Fusobacterium (6.71\%), Neisseria (6.53\%). The abundance of non-atrophic gastritis group was followed by the Prevotella (20.39\%), Neisseria (9.7\%), Alloprevotella (8.69\%), Streptococcus (7.28\%), Fusobacterium (6.9\%). Further analysis revealed that the relative abundance of Neisseria and Aggregatibacter in atrophic gastritis group were significantly lower than that in control group $(P<0.05)$ (Figure 4$)$.

\subsection{Functionally Predicted Metabolic Pathways Underlying the Two Groups}

A total of 24 KEGG metabolic pathways were identified to show significantly different microbiota abundances between the two groups (Figure 5). The metabolic pathways significantly upregulated in atrophic gastritis group at level 3 were enriched in: taurine and hyposulfonic acid metabolism, ribonucleic acid polymerase, fructose and mannose metabolism, starch and sucrose metabolism, glycolysis/gluconeogenesis, etc. $(P<0.05)$. Other pathways including glucose biosynthesis, metabolism, $\alpha$-linoleic acid metabolism, glyoxylate and dicarboxylate metabolism $(P<0.05)$, had more abundance in the control group than in the atrophic gastritis group.

\section{Discussion}

The oral cavity is connected with the respiratory system, the digestive system and the external environment, which provides suitable environment and conditions for the growth, reproduction and settlement of various microorganisms in the oral cavity, thus forming a complex oral microecological environment. The coating on the tongue is a thin, white and moist coating on the back of the tongue, mainly composed of desquamated epithelial cells, blood cells, metabolites, nutrients and bacteria, where is the main distribution center of oral flora [19]. In recent years, with the development of genomics, new achievements have been emerging in the research on the relationship between tongue coating microbiota and disease.

It has been found that the tongue microbiota is of great significance for oral
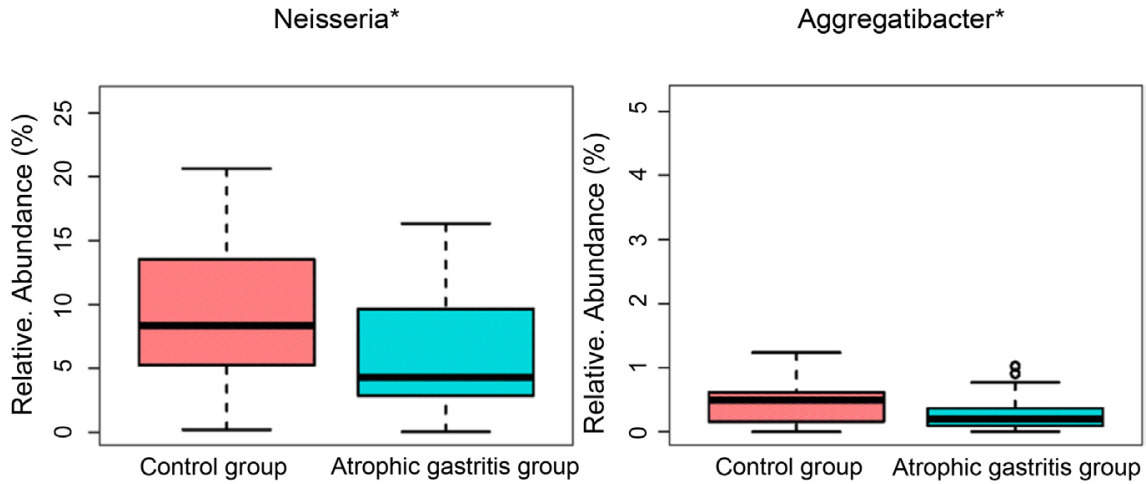

Figure 4. Relative abundance of Neisseria and Aggregatibacter in two groups. 


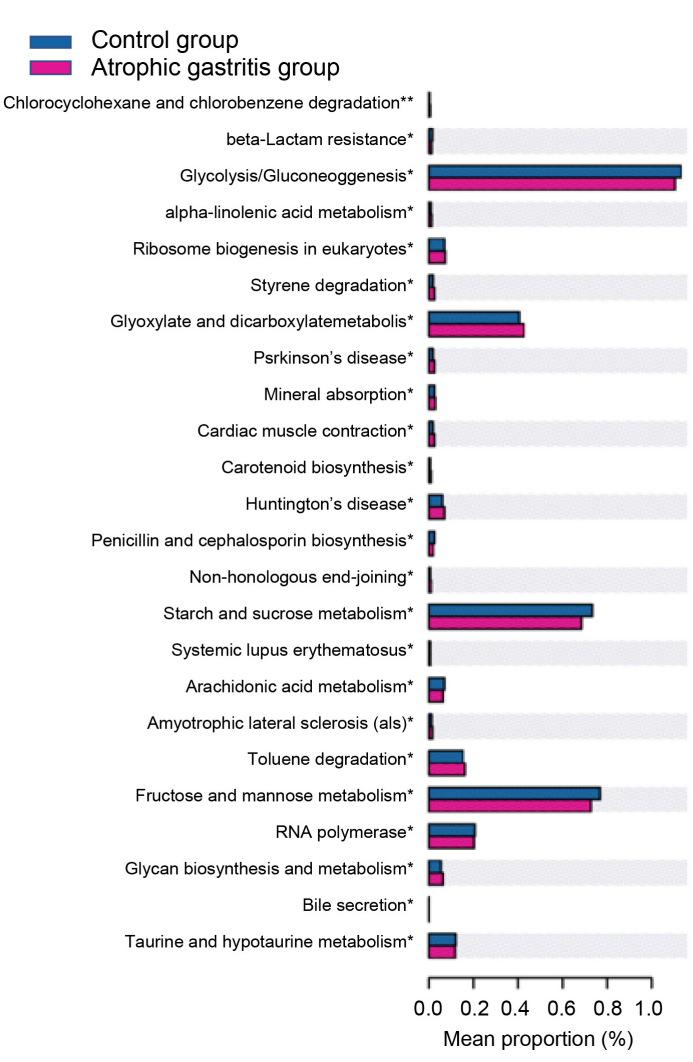

$95.0 \%$ confidence intervals

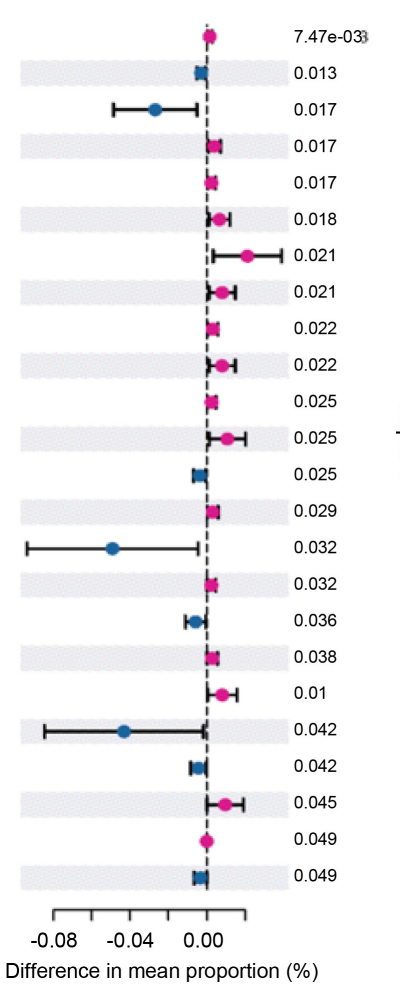

Figure 5. Metabolic function analysis of tongue coating flora in two groups.

health, and the imbalance of oral microecology may lead to caries [20], halitosis [21], periodontitis [22] and other oral diseases. In addition, the imbalance of tongue microbiota may directly involve in the occurrence of diseases beyond the oral. Study has demonstrated a significant association between the Porphyromonas gingivalis and rheumatoid arthritis disease, which suggests that improving oral flora may be a new direction to alleviate the symptoms of rheumatoid arthritis and delay the disease progression [23]. Moreover, a large number of candida albicans were found in tongue coating cultures in patients with chronic kidney disease, implying that tongue coating may be one of the portals for disseminated infection [24]. The relative abundance of Neisseria, Haemophilus, Fusobacterium and Porphyromonas in the healthy people were higher than that in the cancers, suggested that tongue diagnosis may provide potential screening and early diagnosis method for cancer [25].

In this study, we used the 16S rRNA gene sequencing technology to analyze the composition and diversity of the tongue coating microbiota and compared them between atrophic gastritis group and non-atrophic gastritis group. Comparing bacterial taxa at each level with the control group, Betaproteobacteria and Spirochaetia were enriched in the non-atrophic gastritis group at the class level. At the genus level, the atrophic gastritis group had lower proportion of Neisseria and Aggregatibacter compared with the control group. Its mechanism may be involved in the local microenvironment inflammation, which in turn indirectly 
promotes the occurrence and development of gastritis. In addition, we used PICRUSt software to predict that these changes of tongue coating flora can cause functional changes in 24 pathways, suggesting that these functional changes may be the cause of the symptoms of atrophic gastritis.

Li et al. [26] found that the abundance of Campylobacter gradually increased during the progression of gastric mucosa from normal to superficial, atrophic gastritis, and intestinal metaplasia, which suggested that tongue coating flora may be expected to become a non-invasive new biomarker suitable for long-term monitoring of gastritis progression. Another study [27] found that the relative abundance of Proteobacteria, Neisseria and Haemophilus in tongue fur of gastric cancer patients were significantly lower than that of healthy patients, indicating the potential of oral microbial detection as a means of early diagnosis or screening of gastric cancer in the future. At present, the diagnosis of atrophic gastritis is mainly invasive endoscopic examination, which causes great pain and high cost for patients. If the change of the microbial community of tongue coating is used as a screening method for patients, it will bring important changes to the early diagnosis of atrophic gastritis.

There were still limitations in this study. The current sample size of this study is still small, and dietary habits, regional environment and other factors were not taken into account. Although the association can be derived from the results of the study, the cross-sectional design does not finally prove causality. Moreover, in this paper, the differences between the bacterial flora of the tongue coating were discussed, but the metabolites and pathogenic mechanisms of the specific bacterial flora were not further analyzed. The interrelation between functional roles of identified bacterial species and atrophic gastritis should be further elaborated in the follow-up studies.

\section{Conclusion}

We investigated that the tongue coating microbiota differed in atrophic gastritis patients compared with non-atrophic gastritis controls by $16 \mathrm{~S}$ rRNA sequencing technology. The composition and diversity of the tongue coating microbiota differ at the class and genus between the two groups and functionally predicted metabolic pathways between the two groups were also discussed in our finding. We provide novel evidence that tongue coating microbiota may be a biomarker for characterizing patient with atrophic gastritis, but its mechanism needs to be further elaborated.

\section{Conflicts of Interest}

None.

\section{Funding Information}

This work is supported by the Wenzhou traditional Chinese medicine construction project (Grant No. 2018008). 


\section{References}

[1] Sharma, A. and Gilbert, J.A. (2018) Microbial Exposure and Human Health. Current Opinion in Microbiology, 44, 79-87. https://doi.org/10.1016/j.mib.2018.08.003

[2] Fernandez, M.F., Reina-Perez, I., Astorga, J.M., Rodriguez-Carrillo, A., Plaza-Diaz, J. and Fontana, L. (2018) Breast Cancer and Its Relationship with the Microbiota. International Journal of Environmental Research and Public Health, 15, 1747. https://doi.org/10.3390/ijerph15081747

[3] Parida, S. and Sharma, D. (2019) The Microbiome-Estrogen Connection and Breast Cancer Risk. Cells, 8, 1642. https://doi.org/10.3390/cells8121642

[4] Rea, D., Coppola, G., Palma, G., Barbieri, A., Luciano, A., Del Prete, P., Rossetti, S., Berretta, M., Facchini, G., Perdona, S., et al. (2018) Microbiota Effects on Cancer: From Risks to Therapies. Oncotarget, 9, 17915-17927.

https://doi.org/10.18632/oncotarget.24681

[5] Mayer, E.A., Knight, R., Mazmanian, S.K., Cryan, J.F. and Tillisch, K. (2014) Gut Microbes and the Brain: Paradigm Shift in Neuroscience. Journal of Neuroscience, 34, 15490-15496. https://doi.org/10.1523/JNEUROSCI.3299-14.2014

[6] Lee, C.J., Sears, C.L. and Maruthur, N. (2020) Gut Microbiome and Its Role in Obesity and Insulin Resistance. Annals of the New York Academy of Sciences, 1461, 37-52. https://doi.org/10.1111/nyas.14107

[7] Song, M.C.A. and Sun, J. (2020) Influence of the Gut Microbiome, Diet, and Environment on Risk of Colorectal Cancer. Gastroenterology, 158, 322-340. https://doi.org/10.1053/j.gastro.2019.06.048

[8] Singer-Englar, T.B.G. and Mathur, R. (2019) Obesity, Diabetes, and the Gut Microbiome: An Updated Review. Expert Review of Gastroenterology \& Hepatology, 13, 3-15. https://doi.org/10.1080/17474124.2019.1543023

[9] Adamu, M.A., Weck, M.N., Gao, L. and Brenner, H. (2010) Incidence of Chronic Atrophic Gastritis: Systematic Review and Meta-Analysis of Follow-Up Studies. European Journal of Epidemiology, 25, 439-448. https://doi.org/10.1007/s10654-010-9482-0

[10] Lahner, E., Conti, L., Annibale, B. and Corleto, V.D. (2020) Current Perspectives in Atrophic Gastritis. Current Gastroenterology Reports, 22, Article No. 38. https://doi.org/10.1007/s11894-020-00775-1

[11] Correa, P. (1995) Helicobacter Pylori and Gastric Carcinogenesis. The American Journal of Surgical Pathology, 19, S37-S43.

[12] Marshall, B.J. and Warren, J.R. (1984) Unidentified Curved Bacilli in the Stomach of Patients with Gastritis and Peptic Ulceration. Lancet, 323, 1311-1315. https://doi.org/10.1016/S0140-6736(84)91816-6

[13] Keszei, A.P.G., Schouten, L.J., Jakszyn, P. and van den Brandt, P.G. (2013) Dietary N-Nitroso Compounds, Endogenous Nitridation, and the Risk of Esophageal and Gastric Cancer Subtypes in the Netherlands Cohort Study. The American Journal of Clinical Nutrition, 97, 135-146. https://doi.org/10.3945/ajcn.112.043885

[14] Sun, S., Wei, H., Zhu, R., Pang, B., Jia, S., Liu, G. and Hua, B. (2018) Biology of the Tongue Coating and Its Value in Disease Diagnosis. Complementary Medicine Research, 25, 191-197. https://doi.org/10.1159/000479024

[15] Engstrand, L.G.D. (2020) Microbiome and Gastric Cancer. Digestive Diseases and Sciences, 65, 865-873. https://doi.org/10.1007/s10620-020-06101-Z

[16] Keku, T.O.D.S., Deveaux, A., Jovov, B. and Han, X. (2015) The Gastrointestinal Microbiota and Colorectal Cancer. The American Journal of Physiology-Gastrointestinal 
and Liver Physiology, 308, G351-G363. https://doi.org/10.1152/ajpgi.00360.2012

[17] Zhang, B.W., Sun, W.L., Yu, N., Sun, J., Yu, X.X., Li, X., et al. (2018) Anti-Diabetic Effect of Baicalein Is Associated with the Modulation of Gut Microbiota in Streptozotocin and High-Fat-Diet Induced Diabetic Rats. Journal of Functional Foods, 46, 256-267. https://doi.org/10.1016/j.jff.2018.04.070

[18] Ravi, R.K.W.K., Khosroheidari, M. (2018) MiSeq: A Next Generation Sequencing Platform for Genomic Analysis. In: DiStefano, J., Ed., Disease Gene Identification, Methods in Molecular Biology, Vol. 1706, Humana Press, New York, 223-232. https://doi.org/10.1007/978-1-4939-7471-9_12

[19] Casemiro, L.A.M.C., de Carvalho, T.C. and Panzeri, H.M.A.L. (2008) Effectiveness of a New Toothbrush Design versus a Conventional Tongue Scraper in Improving Breath Odor and Reducing Tongue Microbiota. Journal of Applied Oral Science, 16, 271-274. https://doi.org/10.1590/S1678-77572008000400008

[20] Belibasakis, G.N. (2018) Microbiological Changes of the Ageing Oral Cavity. Archives of Oral Biology, 96, 230-232.

https://doi.org/10.1016/j.archoralbio.2018.10.001

[21] Bernardi, S.K.L., Filippi, A., Anderson, A.C., Zürcher, A., Hellwig, E., Vach, K., Macchiarelli, G., Al-Ahmad, A. (2020) Combining Culture and Culture-Independent Methods Reveals New Microbial Composition of Halitosis Patients' Tongue Biofilm. MicrobiologyOpen, 9, e958. https://doi.org/10.1002/mbo3.958

[22] Zhu, B., Macleod, L.C., Kitten, T. and Xu, P. (2018) Streptococcus Sanguinis Biofilm Formation \& Interaction with Oral Pathogens. Future Microbiology, 13, 915-932. https://doi.org/10.2217/fmb-2018-0043

[23] Ceccarelli, F., Orru, G., Pilloni, A., Bartosiewicz, I., Perricone, C., Martino, E., Lucchetti, R., Fais, S., Vomero, M., Olivieri, M., et al. (2018) Porphyromonas gingivalis in the Tongue Biofilm is Associated with Clinical Outcome in Rheumatoid Arthritis Patients. Clinical \& Experimental Immunology, 194, 244-252. https://doi.org/10.1111/cei.13184

[24] Pieralisi, N., de Souza Bonfim-Mendonca, P., Negri, M., Jarros, I.C. and Svidzinski, T. (2016) Tongue Coating Frequency and Its Colonization by Yeasts in Chronic Kidney Disease Patients. European Journal of Clinical Microbiology \& Infectious Diseases, 35, 1455-1462. https://doi.org/10.1007/s10096-016-2684-y

[25] Han, S.W., Yang, X., Qi, Q., Pan, Y.F., Chen, Y.C., Shen, J.J., Liao, H.H. and Ji, Z.N. (2016) Potential Screening and Early Diagnosis Method for Cancer: Tongue Diagnosis. International Journal of Oncology, 48, 2257-2264.

https://doi.org/10.3892/ijo.2016.3466

[26] Cui, J.X., Cui, H.F., Yang, M.R., DuM S.Y., Li, J.F., Li, Y.X., LiuM L.Y., Zhang, X.G. and Li, S. (2019) Tongue Coating Microbiome as a Potential Biomarker for Gastritis Including Precancerous Cascade. Protein \& Cell, 10, 496-509.

https://doi.org/10.1007/s13238-018-0596-6

[27] Hu, J., Han, S.W., Chen, Y. and Ji, Z.N. (2015) Variations of Tongue Coating Microbiota in Patients with Gastric Cancer. BioMed Research International, 2015, Article ID: 173729. https://doi.org/10.1155/2015/173729 